\title{
EARLY STRONG INTERACTION COUNTER EXPERIMENTS
}

\author{
Kent M. Terwilliger \\ University of Michigan, Ann Arbor, MI 48109
}

ABSTRACT

The $17^{\circ}$ beam and some $\pi-p$ two body scattering experiments run in the beginning years of the ZGS are discussed.

\section{INTRODUCTION}

I would like in this talk to give you a bit of the flavor of the early counter experiments and their physics results. I will briefly describe the ZGS $17^{\circ}$ beam line where the initial counter type scattering experiments took place, looking at the status of the line at machine turn-on, the equipment available, and the design of the beam for the counter program. This paper will then discuss a few of the early strong interaction counter experiments which turned out to be the start of major research areas at the ZGS. The experiments discussed will be limited to $\pi-p$ two body scattering - most of the initial strong interaction experiments were of this type, and the kaon and proton work will be considered in other papers.

Two $\pi-p$ elastic scattering experiments will be

described. One, a systematic study of $\pi^{ \pm} p$ angular distributions as a function of energy, and the second, $\pi^{-} p$ backward scattering, right at $180^{\circ}$, with a very detailed energy dependence. The elastic scattering field has proven one of the strongest ZGS areas. An early large magnet spectrometer experiment presented here, on associated production, $\pi^{+} \mathrm{p} \rightarrow \mathrm{K}^{+} \Sigma^{+}$, was the start of a major spectrometer program, and was a predecessor of the highly successful Effective Mass Spectrometer. Finally, two experiments will be described that began ZGS polarization physics: measurements of the asymmetry parameter in $\pi p$ elastic and charge exchange scattering using a polarized proton target.

\section{$17^{\circ}$ BEAM}

Experiments at the ZGS were first done using an internal target. Three main beams were planned for at turn-on: the $7^{\circ}$ separated beam for the $30^{\prime \prime}$ MURA bubb1e chamber, the $17^{\circ}$ beam for unseparated pion counter experiments, and the $30^{\circ}$ beam for low momentum kaon physics. A layout for the initial stages of the three beams, which obviously coupled closely with the accelerator and shield wall, was worked out quite early, and gave the experimenters a weil defined starting point for the detailed beam design required for their set ups. A schematic of the internal target region and planned initial stages of the three 


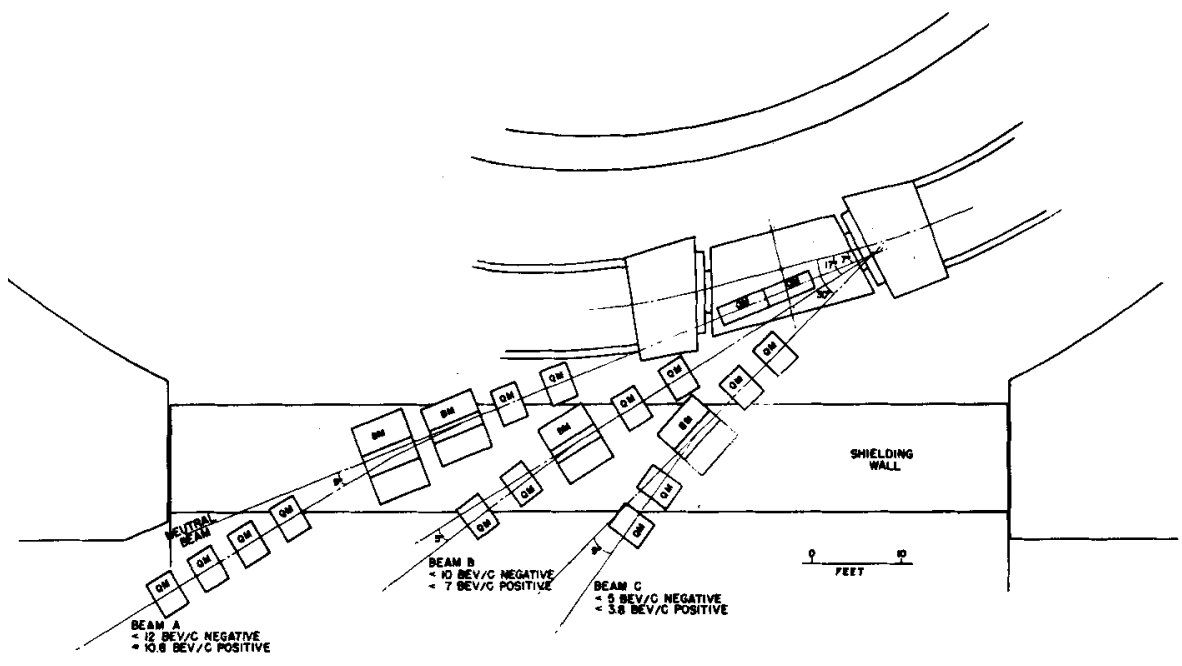

Fig. 1. Plans for internal target area and first stages of beam lines.

beams is shown in Fig. 1. Looking back over the ZGS user group meeting reports, I noted considerable discussion of the expected flexibility of the shield wall and beams - in fact some support posts originally in the design were removed to keep the area open for beam line changes. Once in position, however, the initial beam components became rather permanent - the basic three charged beam line arrangement remained for the life of the internal target, and only the $30^{\circ}$ shield wall section had a major reworking. The possible particle momenta for each beam, determined by the machine field and target position, were calculated by Larry Ratner and were readily available in the ZGS Users Handbook. Fig. 2 is a page from the Handbook showing the

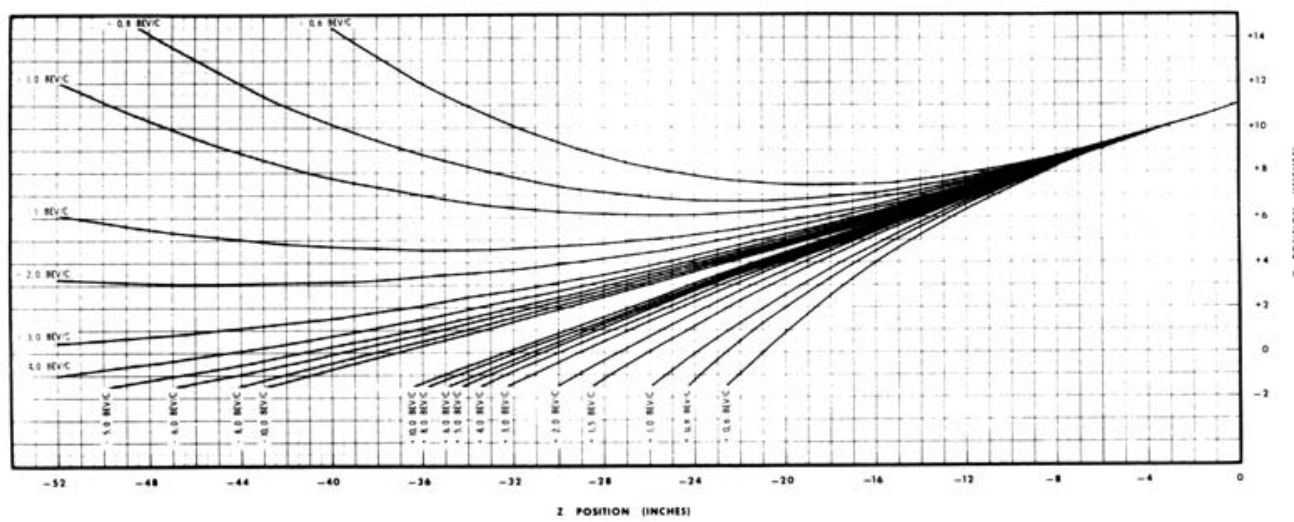

Fig. 2. Allowed momenta for $17^{\circ}$ beam. 


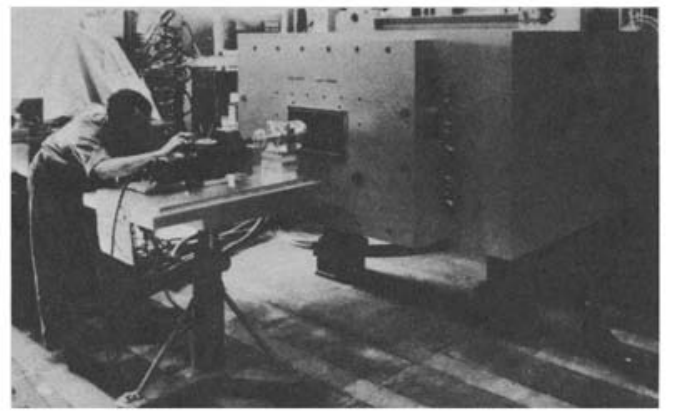
The magnets and quadruples were well measured and were standard enough that experimenters could generally take them as given units for a beam, and quite reliably design the beam and predict its properties. From the experimenters' sketches the ZGS staff would make layout

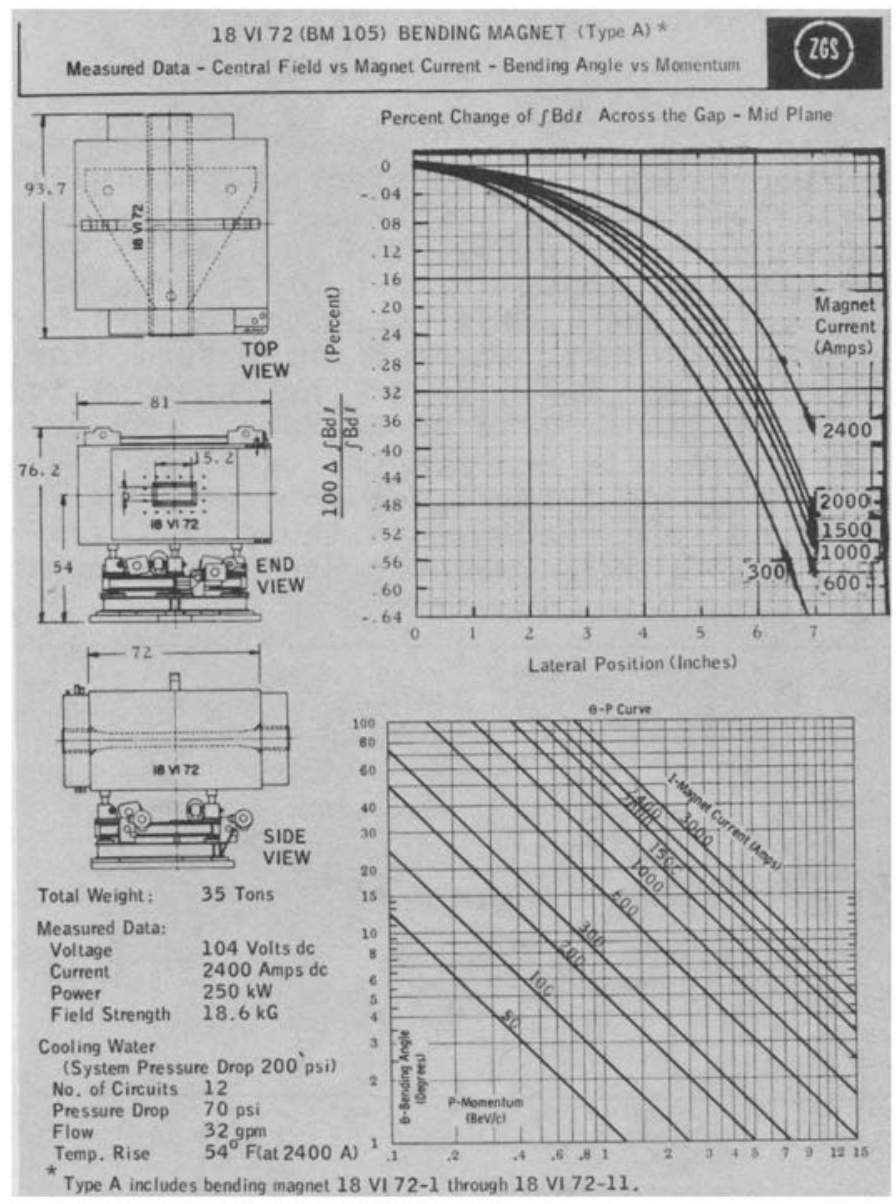

Fig. 4. BM105 properties.

$17^{\circ}$ beam range. Also available for initial experiments was an adequate supply of bending magnets and quadrupoles. Fig. 3 shows a standard ZGS bending magnet, BM105, being measured and Fig. 4 its properties.

Fig. 3. Bending magnet BM105. 


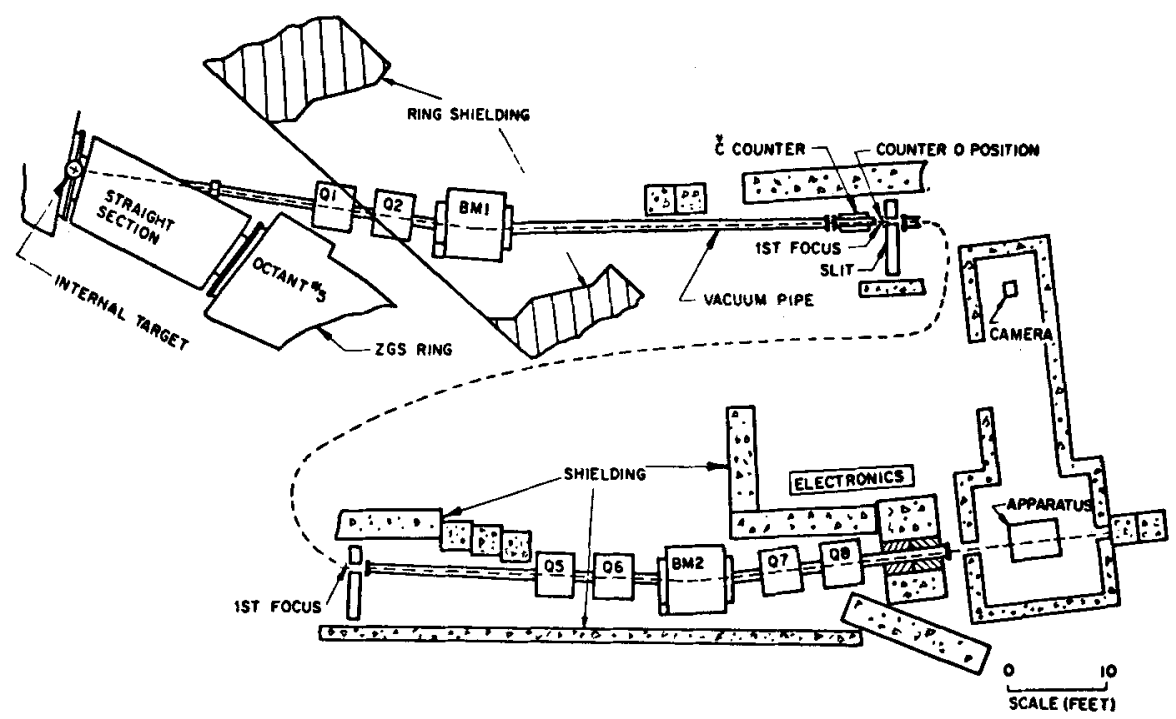

Fig. 5. Layout of $17^{\circ}$ beam and set up of optical spark chamber elastic scattering experiment.

drawings and position the magnets and shielding. An experimenter could rely on top quality professional help right at the start of the ZGS. For the $17^{\circ}$ beam Wayne Nestander's layout drawings with small specified positioning errors materialized on the floor within specs. The $17^{\circ}$ beam we worked up was quite simple a two stage beam (Fig. 5) with $\sim 1 \%$ momentum resolution, about $2 \rightarrow 6 \mathrm{GeV} / \mathrm{c}$, delivering up to $\approx 3 \times 10^{5}$ per $5 \times 10^{11}$ accelerated protons; the $\pi^{+}$intensity, with non $-0^{\circ}$ production, was much lower.

\section{EXPERIMENTS}

\section{ELASTIC $\pi p$ SCATTERING}

$\pi^{ \pm}$p Elastic Scattering $2.3 \rightarrow 6 \mathrm{GeV} / \mathrm{c}(\mathrm{E}-3)$

This Michigan experiment ${ }^{I}$ used optical spark chambers and was designed to be as simple as possible for the initial turn on - measuring elastic scattering in the horizontal plane. The general layout is shown in Fig. 5 . A top view of the spark chamber and a photo of spark chamber tracks of an event are presented in Fig. 6. The experiment was just a bit too simple it turned out four gap chambers are pretty minimal. We also had just two views of each chamber at $90^{\circ}$ which made it hard to handle more than one track, limiting the data rate. A1so some anticoincidence counters could have suppressed 

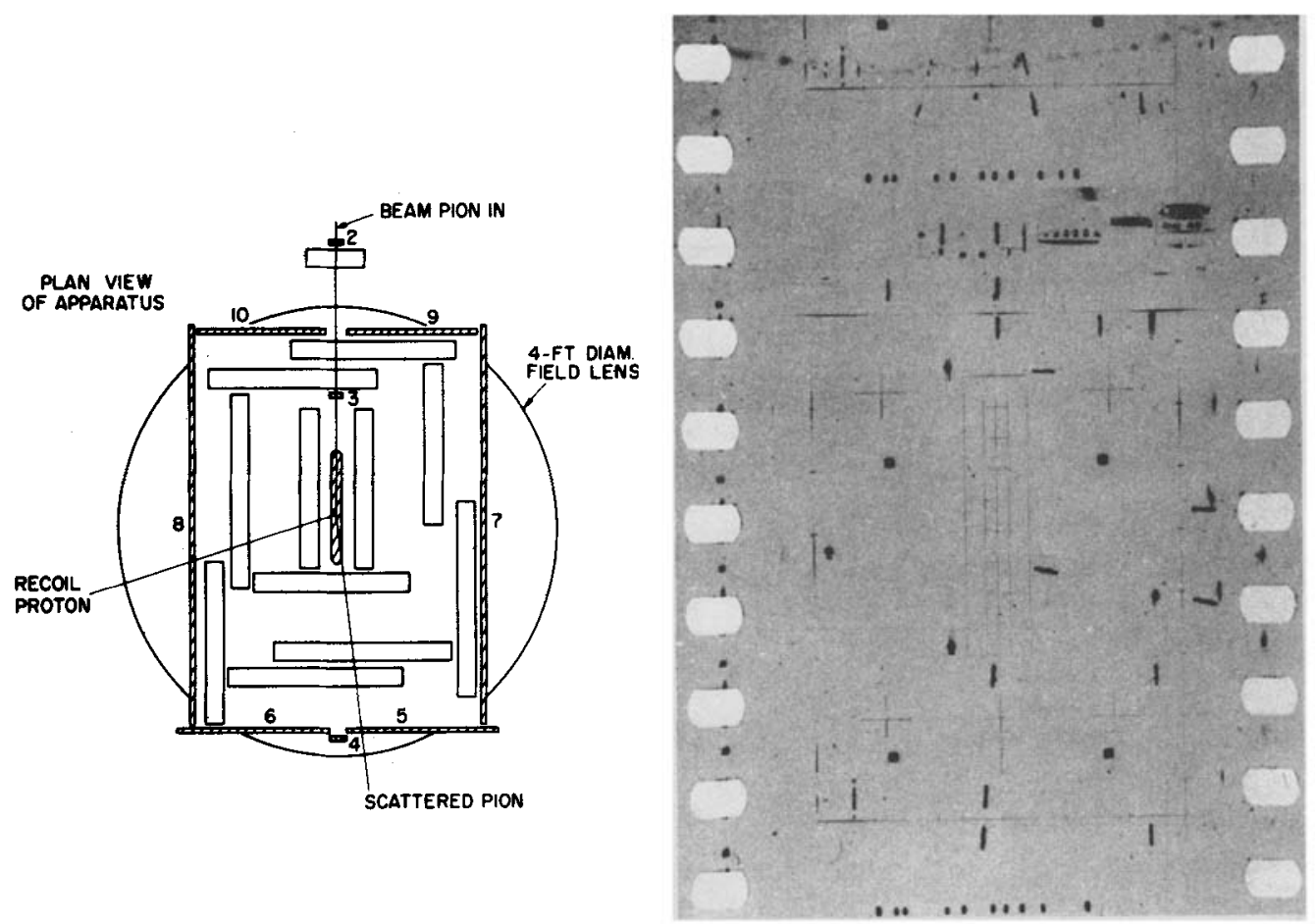

Fig. 6. Left, plan view of spark chambers, right, photo of an event, elastic $\pi^{ \pm} p$ scattering experiment.

many of the inelastics. So, we learned. But since the physics was simple, too, we got reasonable data which rather surprisingly had not yet been obtained elsewhere. Our results, in Fig. 7 and 8 , show that the structure previously observed at lower energy in $\pi-p$ elastic scattering was in fact quite a systematic thenomenon, having similarities to the structure observed in charge exchange scattering.

$\pi^{-}$p $180^{\circ}$ Elastic Scattering, 1.6 to $5.3 \mathrm{GeV} / \mathrm{c}$. (E-30)

Another elastic scattering experiment, done the following year in the same $17^{\circ}$ beam line by Alan Krisch and his group, 2 is probably one of the most often cited experiments from the ZGS - because the group made a set of precision measurements in a region where the theorists found their mode1s could do a lot of predicting: exactly backwards $\pi p$ elastic scattering. This experiment, like the subsequent experiments of Krisch and his group, is conceptually very simple designed with what looks like more than enough constraints- 

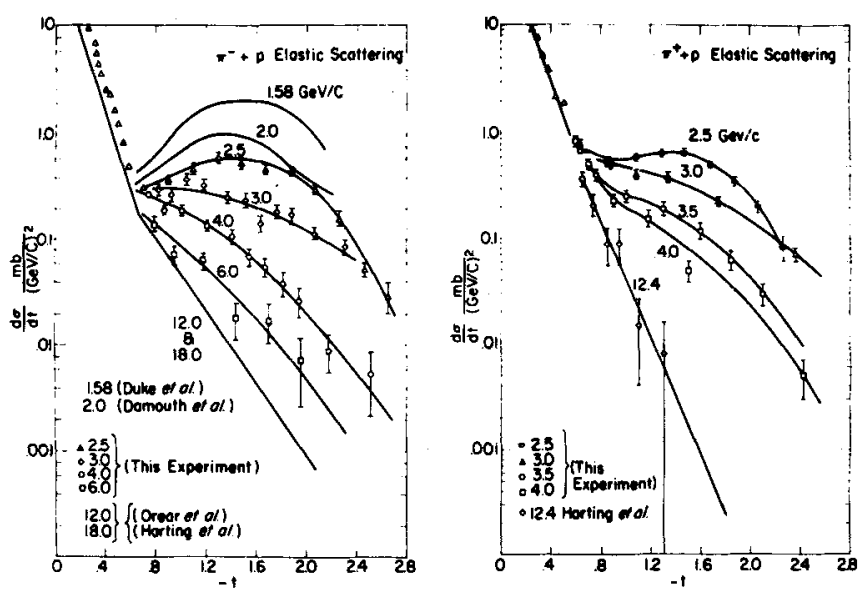

Fig. 7. Energy dependence of angular distributions.
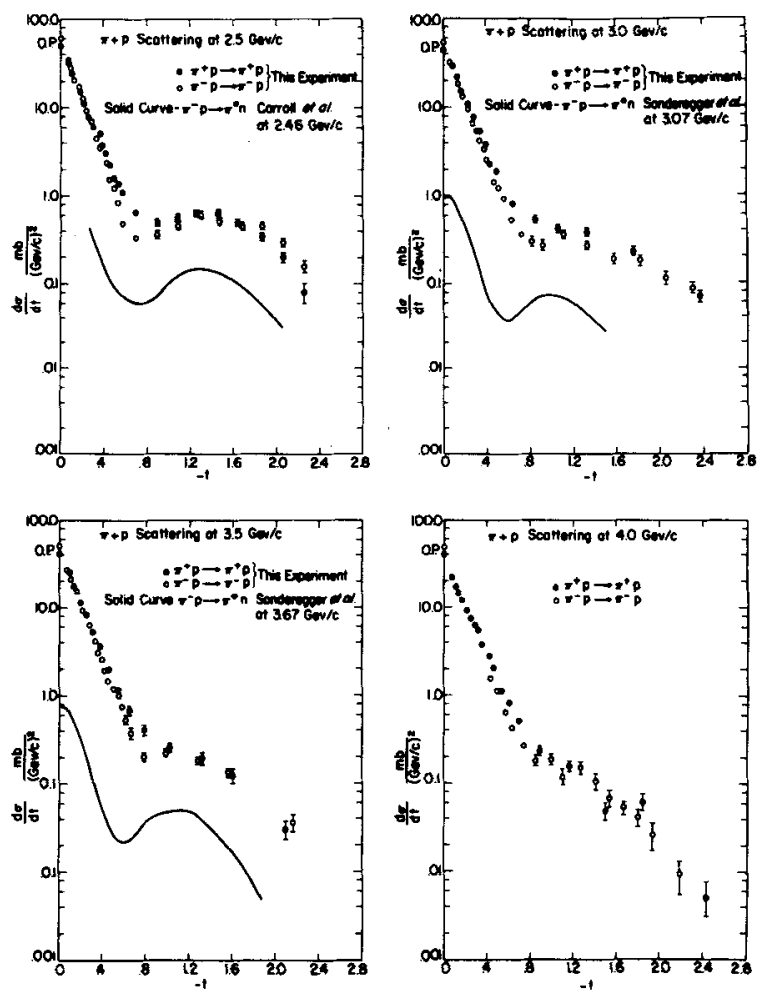

Fig. 8. Comparison with charge exchange scattering. 


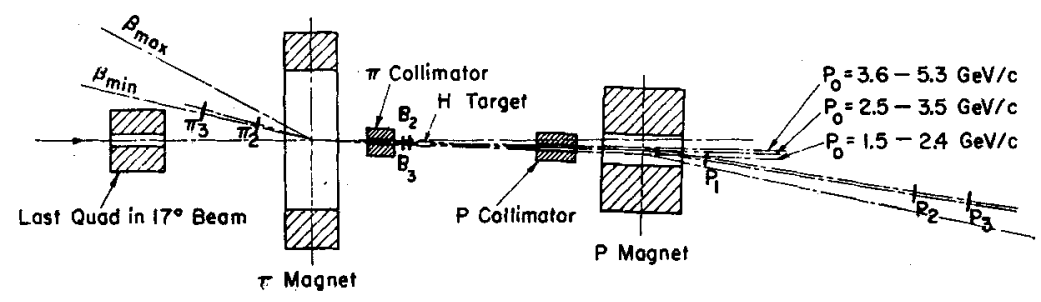

Fig. 9. Layout for backward elastic scattering experiment

an overdesign approach that genera11y pays off, since there are usually problems one hasn't counted on. The group's set up is shown in Fig. 9. With the magnets left stationary they were able to do measurements over a wide range of momenta by just changing fields and moving countersa style of operation the group has continued to use. I remember looking at the experiment for the first time I was somewhat startled by the number of 1 ines on the floorthere was one line for each point, and there were clearly a lot of points being planned. They got their points all right, as seen in Fig. 10. The very rich structure showed strong resonance effects-particularly at the

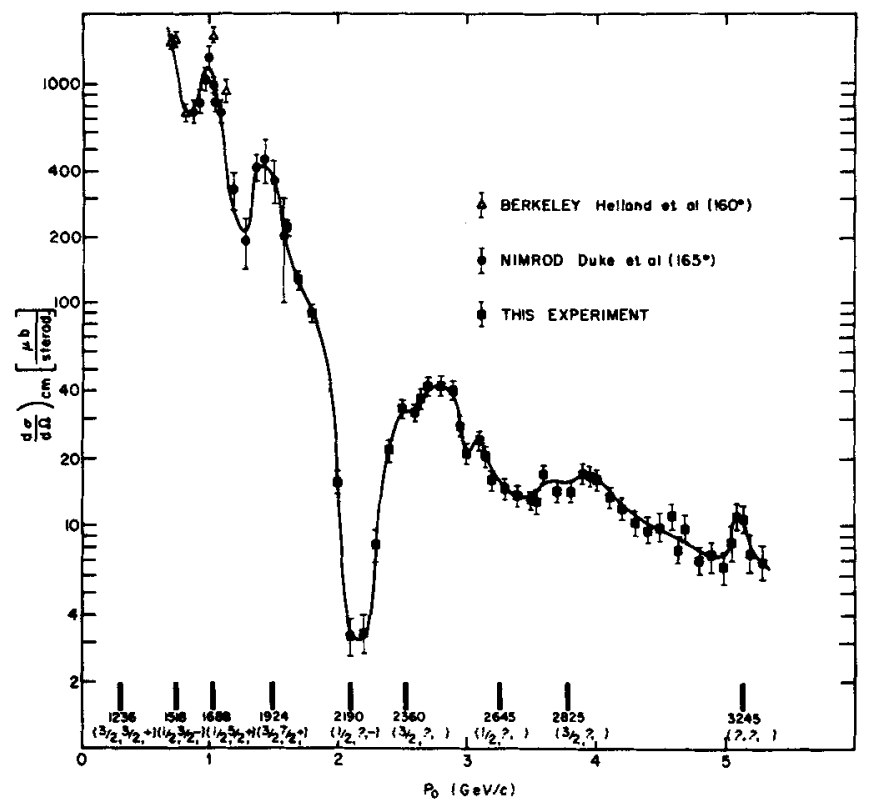

Fig. 10. $180^{\circ} \pi^{-} p$ elastic scattering cross section as a function of $\pi^{-}$laboratory momentum. Resonance positions are indicated. 
center of mass energy of $2190 \mathrm{MeV}$. The destructive or constructive interferences with background enabled many quite clear parity assignments. The couple of standard deviation peak at $3245 \mathrm{MeV}$ hasn't been seen by others though nobody else has looked at exactly $180^{\circ}$ with better statistics.

These two experiments were the first in a series of very productive elastic scattering experiments involving a variety of groups - it has been a strong program.

\section{SPECTROMETER EXPERIMENT}

Very early, wel1 before ZGS turn on, Don Meyer was pushing for the construction of a general purpose spectrometer magnet for spark chamber experiments. 3 The final resulting magnet, SCM105, has turned out to be extreme1y useful (as in the previous described experiment). A schematic of the magnet, from the ZGS Users Handbook, is shown in Fig. 11. This magnet was used by Meyer and his collaborators for a very productive series of spark chamber experiments. I consider that the Effective Mass Spectrometer was at least in part an extension of
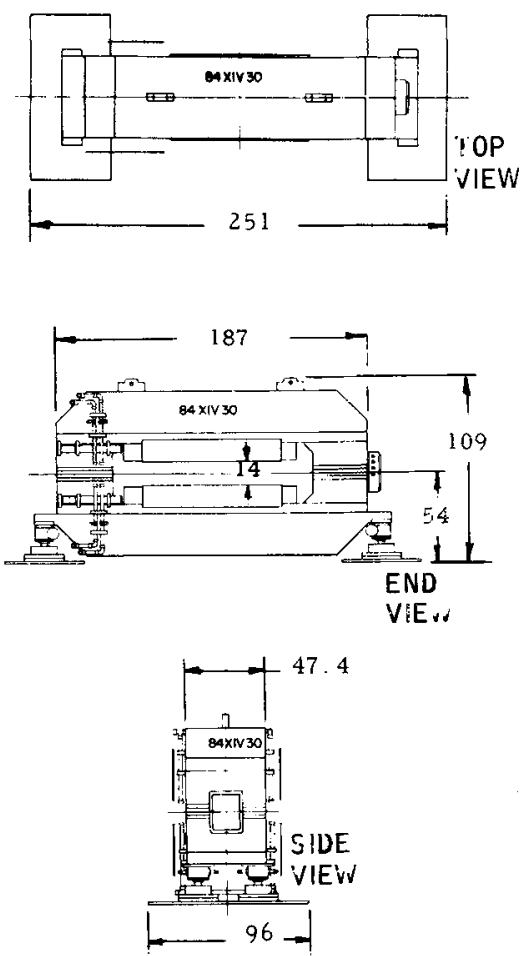

Fig. 11. Dimensions of the SCM105, a general purpose spectrometer magnet. 


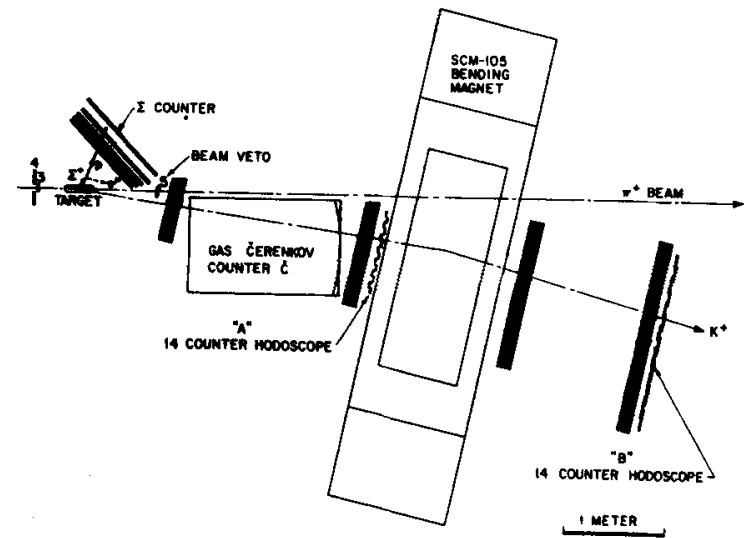

Fig. 12. Plan view of the wire spark chamber experiment $\pi^{+} \mathrm{p} \rightarrow \mathrm{K}^{+} \Sigma^{+}$.

(a)
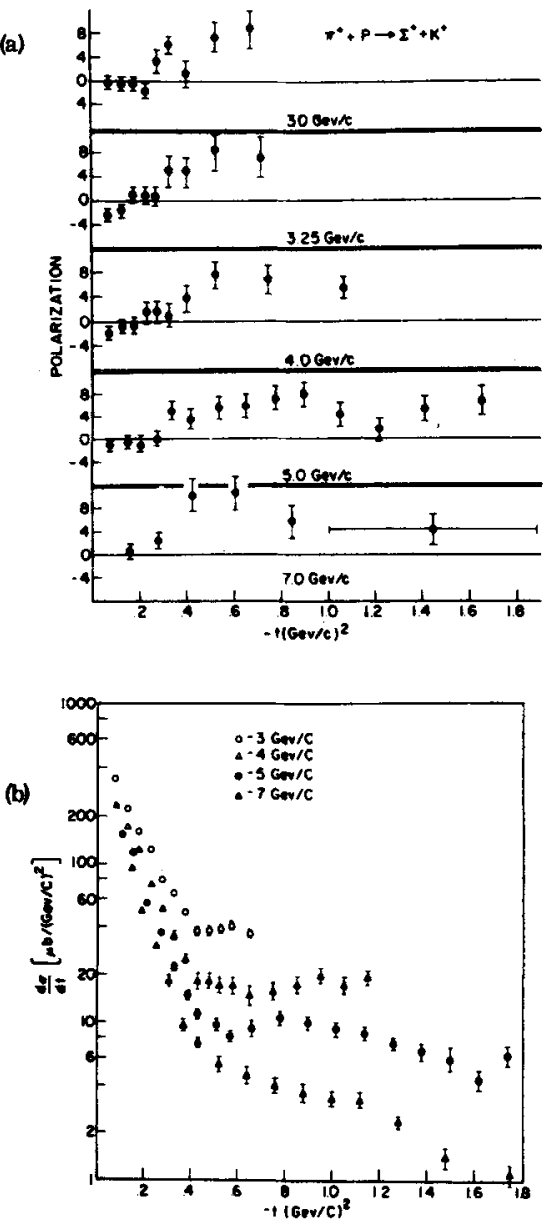

Fig. 13. $\pi^{+}+\mathrm{p} \rightarrow \mathrm{K}^{+}+\Sigma^{+}$experiment.

(a) Polarization of the $\Sigma^{+}$.

(b) Differential cross section. their experimental approach.

$\pi^{+}+\mathrm{p} \rightarrow \mathrm{K}^{+}+\Sigma^{+}, 3$ to $7 \mathrm{GeV} / \mathrm{c}$ (E147)

Their initial experiment 4 was in the External Proton Beam. Their layout is shown in Fig. 12. The forward $\mathrm{K}^{+}$momentum and angle determined the missing mass; the events in the $\Sigma^{+}$peak were further selected by looking at the proton from the $\Sigma^{+}$decay. The up-down asymmetry of the decay protons enabled the determination of the $\Sigma^{+}$ polarization. Their results are shown in Fig. 13. They saw a dramatic change in the slope of the differential cross section going from the diffraction region to $-t \geq .4$ $(\mathrm{GeV} / \mathrm{c})^{2}$, and an apparentiy associated increase in $\Sigma^{+}$ polarization. This cross section and polarization behavior is not unlike that seen in pp scattering; a particularly interesting result for those looking at spin effects.

\section{POLARI ZED TARGET EXPERIMENTS}

The study of polarization physics at the ZGS was started by Aki Yokosawa and his collaborators. 


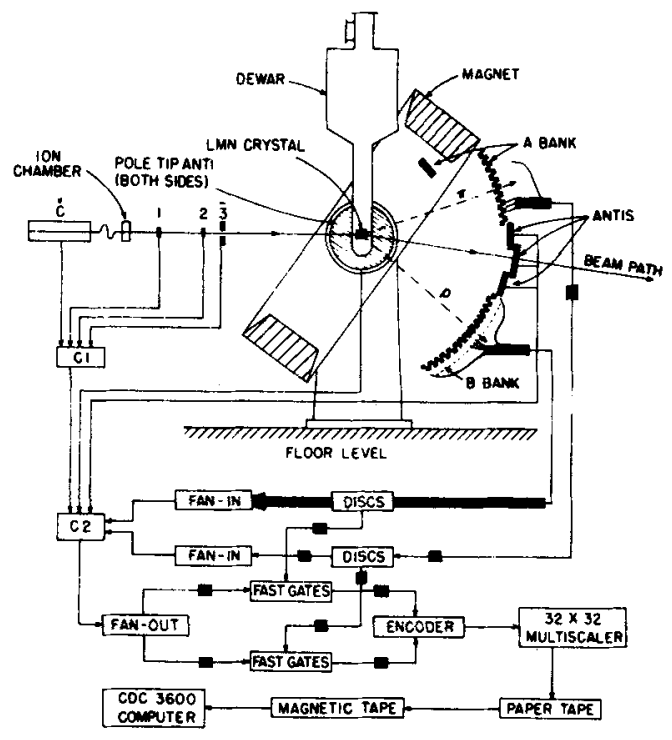

Fig. 14. Schematic of apparatus for $\pi p$ elastic scattering polarization experiment.

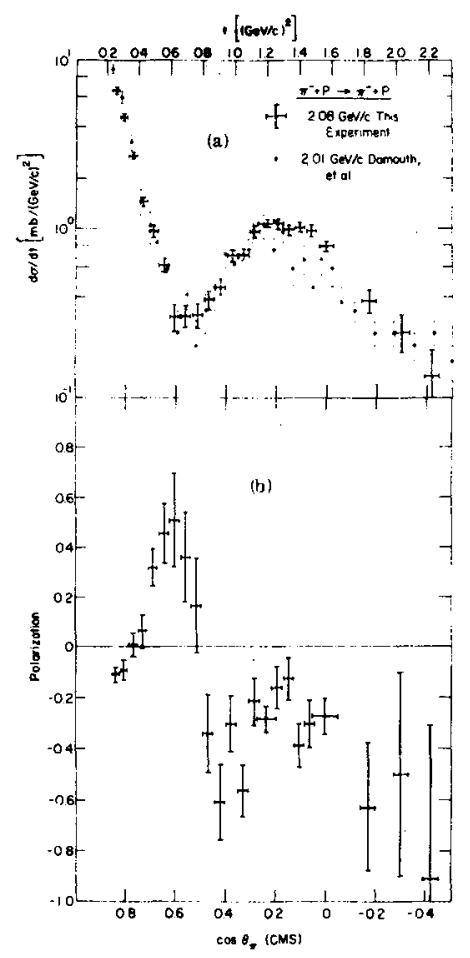

Fig. 15. $\pi^{-} \mathrm{p}$ elastic scattering $2.07 \mathrm{GeV} / \mathrm{c}$. a) Differentia1 cross section. b) Asymmetry parameter.
Their first two polarized target experiments, carried out in the $17^{\circ}$ beam, are discussed here.

Polarization in $\pi^{-} \mathrm{p}$ Elastic Scattering, 1.7 to $2.5 \mathrm{GeV} / \mathrm{c}$ (E17)

The setup for their $\pi^{-} p$ elastic experiment 5 is shown in Fig. 14 . In these first experiments they had to cope with a large background from the LMN polarized target ( $97 \%$ non-hydrogen) a $50 \%$ background subtraction was required in this experiment. Their target polarization was $\sim 50 \%$. Even with these limitations they obtained quite good data, as seen in Fig. 15 . Data of this type over a range of energies enabled the group to do a phase shift analysis, with results shown in Fig. 16. As indicated, they were able to establish the spin and parity of the resonance at 2.07 $\mathrm{GeV} / \mathrm{c}(2190 \mathrm{MeV})$ to be $7 / 2$. Clearly polarization was a powerful tool.

Polarization in $\pi^{-} p \rightarrow \pi^{\circ} n$, $2 \rightarrow 5 \mathrm{GeV} / \mathrm{c}$ (E 111)

Their second polarized target experiment, polarization in charge exchange scattering, 6 required a considerable increase in complexity, as evident in their layout in Fig. 17. With neutron time of flight information 

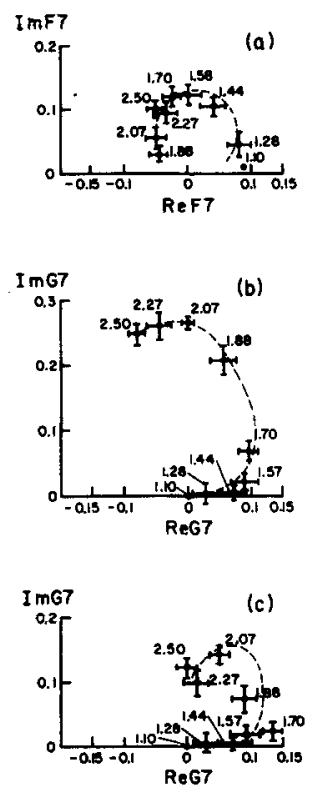

$F_{16}, 16$. The partial-wave amplitudes (a) $A_{1}$ and (b) $A_{1}$ obtained by starting from lower energy solutions and using a continutty condition. (c) The partialwave amplitude $A_{1-}$ obtained by starting from an optical mode.
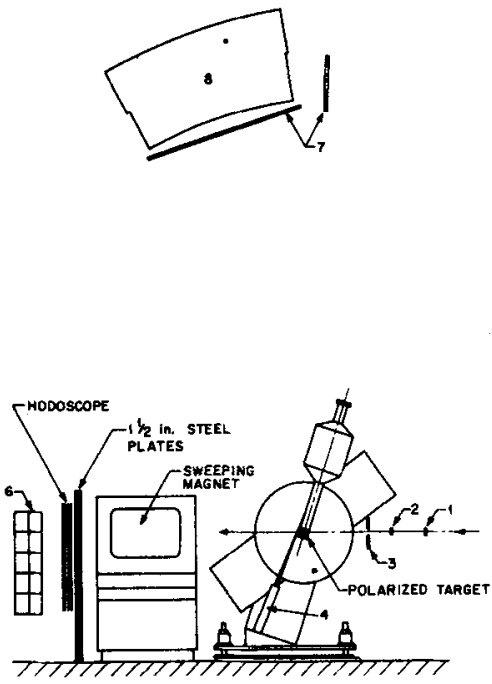

FiG, 17. Side elevation of the experimental arrangement. (1)-(4) are plastic scintillation counters with respective dimensions of 1 in. $\times 1$ in., 1 in. $\times 1$ in., $10 \mathrm{in} \times 10 \mathrm{in}$. with a 1-in. $\times 1$-in. hole, and $3.5 \mathrm{in.}$ $x 8$ in. The lead-Lucite Cherenkov counter (6) consists of ten modules, each 8 in. $\times 16$ in. $\times 4$ in. Anticoincidence counters (7) protect the 52 recoil-neutron counters (8). they obtained a clear signal above background of $\pi^{\circ}$ from the polarized proton. Their polarization data and fits to $J=(\ell \pm 1 / 2)$ predictions from an interference model are shown in Fig. 18. They were ab1e to make a choice for many of the resonances. Yokosawa and his group initiated the ZGS polarization physics work with these two experiments. They have continued this research line through the lifetime of the ZGS with very dramatic physics results.

In closing, I want to say that Roger Hildebrand's vision and determination to have the ZGS wide open to outside users obviously worked out well, as indicated by the experiments I've described: Michigan, one of the universities most heavily involved with MURA, has been able to be a major ZGS user.

This work has been supported by the U.S. Department of Energy. 


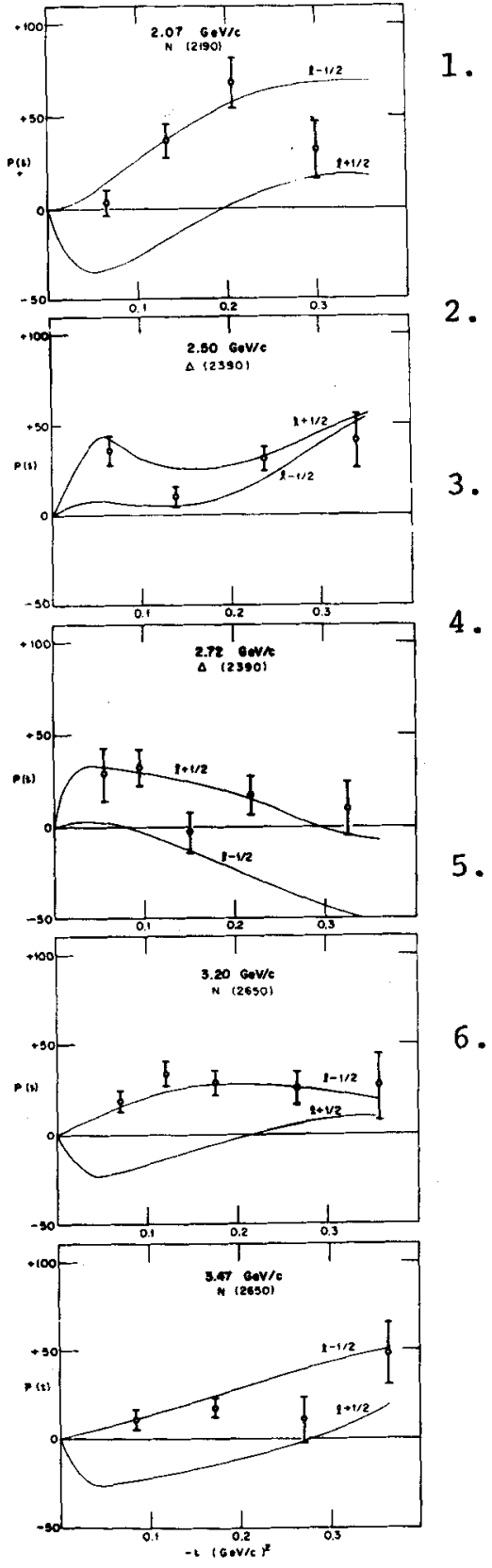

Fig. 18. Polarization in $\pi \mathrm{p}+\pi^{\circ} \mathrm{n}$ and fits to $\mathrm{J}=$ $(\ell \pm 1 / 2)$ predictions.

\section{REFERENCES}

C.T. Coffin, N. Dikmen,

L. Ettlinger, D. Meyer,

A. Saulys, K. Terwilliger, and D. Williams, Phys. Rev. Lett. 15,838 (1965); Ibid $17,45 \overline{8}$ (1966); Phys. Rev. 159, 1169 (1967).

S.W. Kormanyos, A.D. Krisch, J.R. O'Fallon, K. Ruddick, and L.G. Ratner, Phys. Rev. Lett. 16, 709 (1966); Phys. Rev. $\overline{16} 4,1661$ (1967).

D.I. Meyer, Argonne Accelerator Users Group Meeting, Argonne

National Laboratory, Nov. 1962, p. 17 (unpublished).

S.M. Pruss, C.W. Akerlof,

D.I. Meyer, S.P. Ying,

J. Lales, R.A. Lundy, D.R. Rust, C.E.W. Ward, and D.D. Yovanovitch, Phys. Rev. Lett. 23, 189

(1969); Phys. Lett. 30B, 289, (1969).

5. S: Suwa, A. Yokosawa, N. Booth, R. Esterling, R. Hill, Phys.

Rev. Lett. 15, 560 (1965); Phys. Rev. Lett. 16, 714

(1966); Phys. Rev. Dl, 729 (1970).

D.D. Drobnis, J. Lales,

R.C. Lamb, A. Moretti,

R.C. Niemann, T.B. Novey,

J. Simanton, A. Yokosawa, and D.D. Yovanovitch, Phys.

Rev. Lett. 20, 274 (1968);

Phys. Rev. Lett. 20, 353 